MASS DISTRIBUTION AND LUMINOSITY FUNCTION

OF WHITE DWARFS

\begin{abstract}
Volker Weidemann and Jie W. Yuan
Institut $f$. Theoretische Physik u. Sternwarte der Universität Kiel, D-2300 Kiel l, F.R. Germany
\end{abstract}

\title{
1. The white dwarf mass distribution
}

Ever since Graham's Strömgren photometry (1972) demonstrated the existence of a single well defined cooling sequence of DA white dwarfs the question of the mass dispersion (or the width of the number-mass distribution) has been in the foreground of my studies (Weidemann, 1970, 1977).

Indeed it turned out that the shape of the white dwarf mass distribution provides strong constraints on the theory of stellar evolution with mass loss, a fact which will be demonstrated again in the following lecture. It therefore seems worthwhile to dwell in some detail on the methods of its determination. For the benefit of the non-specialists I shall first present some of the historical results and then continue to discuss the present situation.

After my demonstration (1970) that Strömgren photometry was highly superior to Johnson (UBV) photometry, due to the fact that the narrow bands are essentially line-free, and after understanding the s-shape of the line-free DA-sequence as caused by the effects of Balmer depression and H minus opacity as compared to black-body energy distribution it was possible to use calculated two-color diagrams for the determination of surface gravity, with the highest sensitivity around $12000 \mathrm{~K}$. It turned out that the g-distribution - and via the mass-radius relation therefore also the mass distribution - is fairly narrow, around $M \approx 0.6 \mathrm{M}_{\odot}$. observationally, the next step was the use of Oke's multichannel spectrophotometer with which Greenstein secured reliable energy distributions for hundreds of white dwarfs at the Hale $5 \mathrm{~m}$ telescope (see Greenstein, 1976, 1984).

The Kiel group - to which Greenstein kinaly provided most of his observations - was able to evaluate the data, and it turned out that schulz's idea to bin the monochromatic fluxes into wider filters reduced observational and instrumental scatter and thereby provided more reliable g-determinations (see Koester, Schulz, Weidemann, 1979, Fig.6).KSW used 
a weighted least square method to incorporate Strömgren and Johnson colors and Balmer line data, as available, and thus were able to weight their results. Aside from mass distributions derived from surface gravity they also determined radii for stars with known distances and thus obtained mass distributions for $M(R)$.

The difference in the shape and width of the distributions obtained was demonstrated in Fig. 7 to 10 of $\mathrm{KSW}$. The "most" reliable distribution showed a shape which was expected from synthetic calculations for stellar evolution with mass loss as I had shown a few years earlier (Weidemann, 1977, Fig. 2), using a Salpeter initial mass function (IMF) and semiempirical relations between initial and final mass, derived from white dwarf in open clusters of known age or calculated by stellar evolution with wind mass loss according to the Reimers formula.

By variation of IMFs, star formation histories in the Galaxy, and initial-final mass relations Koester and Weidemann (1980) demonstrated how one is able to constrain these essential parameters for models of galactic evolution and estimates of the mass given back to the interstellar medium by the shape of the white dwarf mass distribution. It therefore seemed worth every effort to improve its empirical determination. That this was still necessary could be seen e.g. by the non-agreement of $M(g)$ and $M(R)$ determinations in KSW (Fig.ll).

We thus embarked on the evaluation of the remaining multichannel spectra and concentrated on the most g-sensitive temperature range $16000>T_{\text {eff }}>$ $>8000 \mathrm{~K}$ with improved model atmospheres and least square fits to the whole energy distribution (Weidemann, Koester, 1984) (WK 84). A specific goal was the determination of better parameters for the $\mathrm{zz}$ ceti stars. The resulting mass distribution for 70 DA stars is reproduced in Fig. 1 . It seems still the most reliable one up to the present time.

Before we go on with its discussion let us consider results obtained for non-DA stars.

For the DB stars Oke's multichannel observations were evaluated in Kiel by the same methods. The results were published by Oke et al. 1984 (OWK) and for the first time demonstrated convincingly that the masses of DA and $D B$ stars are very similar. The same holds for the cooler non-DA stars of type $D C$ and $D Q$, for which masses were determined from effective temperatures obtained with new model atmospheres and from radii in case of known distances. The resulting distributions are shown in Fig. 2 (reproduced from Weidemann, 1987a). 


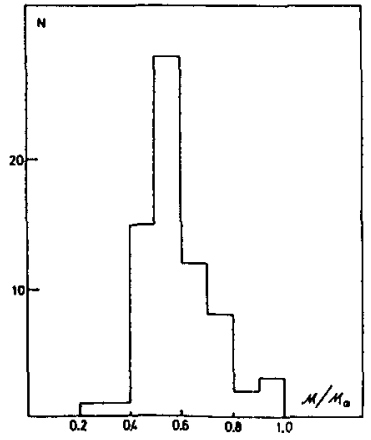

Fig. 1. Mass distribution for $70 \mathrm{DA}$ stars, $\mathrm{M}(\mathrm{g})$. WK 84

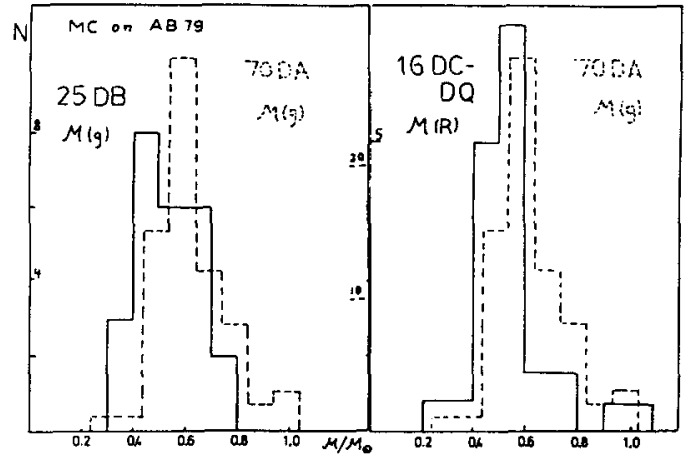

Fig.2. Mass distribution for non DA stars. ---: DA stars for comparison (see text).

A side remark is necessary which at the same time is important for future improvements. Oke's multichannel data were calibrated on AB 79 (Oke and Gunn, 1983) whereas the DA studies used the earlier calibration of Hayes and Latham (1975).

$A B 79$ seemed superior in the DB case as demonstrated by Fig. 2 and 6 in OWK. If so the DA results should also be corrected. The difference amounts to an average increase of about $0.04 \mathrm{M}_{\odot}$ as compared to KSW and WK 84. This implies that the average mass of DA's goes up to $0.62 \mathrm{M}$ from $0.58 \mathrm{M}_{6}$. The average masses are compared in Table 3 of Weidemann, $1987 \mathrm{a}$. We may thus conclude that the non-DA masses could in the average be somewhat smaller, although this seems not to be significant. The recent study of the kinematics of DA and DB stars by Sion et al. (1988) also reaches the conclusion that there are no significant differences between both spectral types.

Sion et al. determined masses $M(R)$ via radii from effective temperatures and distances. I took their Fig. 3 and compare both histograms (normalized to equal area). Although the temperature ranges show little overlap (13000-16000 K) the similarity is striking, and the distribution appears even somewhat narrower than the one of WK 84 . However we may not fall into a trap: Sion et al. derived the masses from radii, $M(R)$ with the $M-R-r e l a t i o n$, with radii from $T_{\text {eff }}$ and distances using single valued $T_{\text {eff }}(c o l o r)$ and $M_{v}$ (color) relations (from Shipman, 1979 and Greenstein, 1984), where the first is calculated for $\log g=8\left(\approx M=0.58 M_{\Theta}\right)$ and the second from an empirical mean fit to observational data for stars with known parallaxes.

This implies to assign to a given color an average radius which corresponds of course to the average mass. With other words: had sion et al. not included many stars with independently determined $T_{\text {eff }}$ and $g$ or $M(R)$ or $\mathrm{M}_{\mathrm{v}}$ from parallaxes the distribution would only reflect the deviation 
of the empirical $M_{V}$-color relation (e.g. Greenstein 1984, 1985) from a calculated $\mathrm{M}_{\mathrm{V}}$-color relation for $\mathrm{M}=\langle\mathrm{M}\rangle$. The difference is, as expected, minute. Greenstein (1985), however, used his empirical studies of multichannel data to estimate also the width of the distribution and finds with $\sigma\left(M_{v}\right)=0.25$ corresponding to a mass range from $-0.10,+0.14 M_{\theta}$ in essential agreement with WK 84 (see Table 5 of Greenstein, 1985). It is remarkable that this refers to the $M(R)$ distribution of 52 white dwarfs of different spectral types, (omitting a few deviating objects like EG 11, L870-2, which has recently been shown to be a close binary by Saffer et al., 1988). Thus there can be little doubt that the distribution is at least as narrow as shown in Fig. 1.

However, several questions arise at this point:

1) how does this distribution compare with the distribution of progenitors: central stars of planetary nebulae, or core masses of stars leaving the AGB?

2 ) how can one understand the observed distribution in terms of models of galactic and stellar evolution?

3) how to improve the results observationally and theoretically?

We address each question in turn in the next sections.

\section{Comparison with M-distributions of}

white dwarf progenitors.

Today it is generally accepted that the immediate progenitors of white dwarfs are in the large majority central stars of planetary nebulae (CPN). Schönberner (1981) has first demonstrated how one can use evolutionary post-AGB tracks and kinematical ages in order to determine CPN masses in cases where distances are known. The luminosity in the plateau phase equals that of a star with the same core-mass in the AGB phase. The rate of evolution along the tracks is determined by burning of the remaining hydrogen fuel. After its exhaustion follows a fast decline of luminosity. As can be seen from the time marks along the tracks, planetary nebulae (PN) are not excited for CPN with $M<0.55 \mathrm{M}_{\odot}$, since those stars evolve too slowly. In the course of time some exceptions have become apparent, which I shall not discuss, instead I refer to the literature, e.g. to the forthcoming Proceedings of the IAU Symposium No. 101 in Mexico City, Oct. 1987. A survey of the methods has also been given in my Tucson lecture last year (Weidemann, 1987a).

The main results of the Schönberner method (see Schönberner and weidemann, 1983) is a confirmation of the narrow highly peaked M-distribution around $0.6 \mathrm{M}_{\odot}$, (see Fig. 5, below). 
However there are two differences: one expected, one unexpected. The first is the absence of CPN below $0.55 \mathrm{M}_{0}$, the second is the steeper decrease towards the high mass tail. Part of it is certainly due to selection effects which discriminate against high mass CPN which pass the high luminosity phase very quickly, but part of it may be due to the possibility that the white dwarf distribution is in reality also narrower and appears broadened due to observational errors. Indeed, the post-AGB luminosity is so extremely sensitive to mass that a distribution could be determined with comparatively higher accuracy, if only distances of PN were better known, or the ensemble on the plateau phase were more complete. Unfortunately both is not the case, and it appears that progress can be made only if one either goes to extragalactic PN's or concentrates on low luminosity CPN's which are hard to find and to observe.

Both ways have been entered now. Ford et al. (1988) find evidence for a steep high mass tail above $0.6 \mathrm{M}_{\odot}$ with $\sigma \leqslant 0.05 \mathrm{M}_{0}$ by observations of [OIII] luminosities for local group galaxies and comparison with evolutionary calculations, whereas a similar small scatter around $0.6 M_{0}$ has been derived for the Magellanic Clouds. Barlow (1988) combines these MC results to find $(0.597 \pm 0.023) \mathrm{M}_{0}$ for $17 \mathrm{CPN}$.

A different claim was made by Heap and Augensen (1987) which evaluated IUE UV fluxes instead of $M_{v}$ by the schönberner method to derive a broader M-distribution with a maximum at $0.65 \mathrm{M}_{\odot}$. However I have shown (Weidemann, 1988) that the discrepancies completely disappear if larger distances are applied, for which there is other evidence. For details I refer to my paper.

A third possibility to derive a progenitor M-distribution is a comparison of AGB luminosity functions with synthetic evolutionary calculations. This method has been applied to the LMC AGB luminosity function of Reid and Mould (1985). First results were presented at the Calgary Workshop 1986 (Weidemann, 1987b) and a detailed explanation of the method has been published in my recent paper (Weidemann, 1987c).

The results are sensitive to the location of the start-TP-AGB relation in the initial mass-luminosity plane.

If thermal pulses start early - as shown by recent evolutionary calculations - the derived M-distribution of stars leaving the AGB becomes very narrow, intermediate between the extremely small (selection effect dominated) CPN distribution and the comparatively wider white dwarf distribution.

A final possibility lies in the evaluation of IRAS sources the luminosity distribution of which in the Galaxy, according to Habing (1988), peakes at $\log \mathrm{L} / \mathrm{L}_{\odot}=4000$ (corresponding to an $\mathrm{AGB}$ core mass of $0.57 \mathrm{M}_{\odot}$ ). Habing 
concludes that the M-distribution is very similar to that of the white dwarfs which is taken as support for the hypothesis that the IRAS point sources are the immediate predecessors of white dwarfs.

\section{Mass distributions calculated with \\ galactic evolution models}

Yuan (1987a) has updated our galactic evolution program, which considers single pool models specified by a given IMF, star formation rate, SFR(t), total age, and initial-final mass relation. Changes compared to our earlier calculations (Koester and Weidemann, 1980, wK 84) concerned mainly incorporation of newer IMFs and $M_{f}\left(M_{i}\right)$-relations, and normalization to a smaller present white dwarf birth rate of $1 \cdot 10^{-12} \mathrm{wD} / \mathrm{pc}^{3} \mathrm{yr}$, revised downwards by a factor of two in view of the results of Fleming, Liebert and Green (1986).

The variety of $M_{f}\left(M_{i}\right)$-relations is shown in Fig. 3. For the discussion of $M_{f}\left(M_{i}\right)$-relations I refer to my lecture at the Mt. Porzio Workshop (Weidemann, 1987d) and the following publication (Weidemann, 1987c). Resulting white dwarf M-distributions become wider as steeper the relations are and begin at smaller masses for lower values of $M_{f}$ for $M_{i}=1$ (about the galactic turn-off).

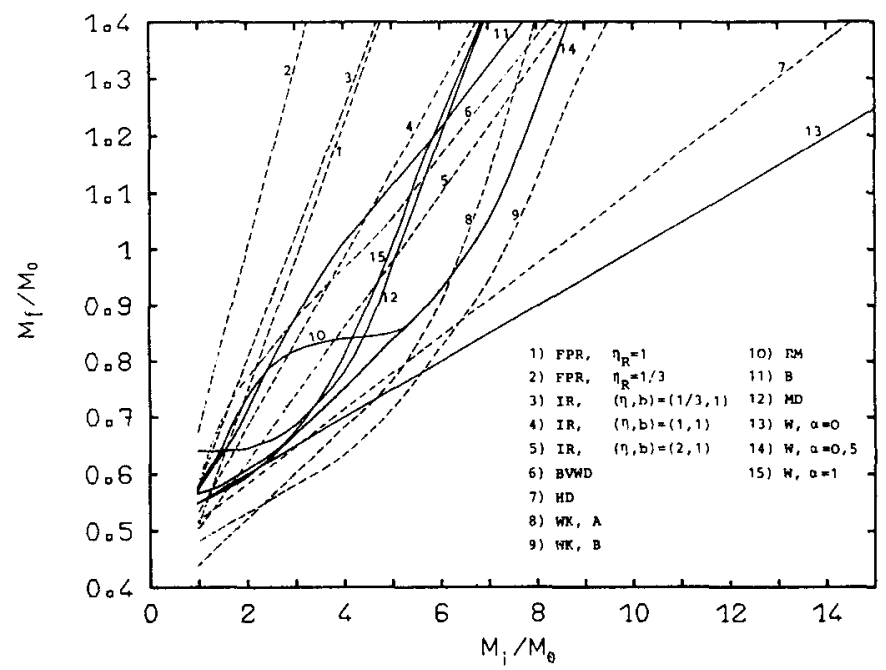

Fig.3. Initial-final mass relations used in galactic evolution model calculations (Yuan, 1987a). 1),2): Fusi-Pecci, Renzini (1976); 3)-5): Iben, Renzini (1983); 6): Weidemann (1977); 7): Hills, Dale (1973); 8),9): Weidemann, Koester (1983); 10): Reid, Mould (1985); 11): Bedijn (1986); 12): Mazzitelli, D' Antona (1987a); 13)-15): Weidemann (1987d). 
Relations 8), 9) and 13) to 15) are based on observations of white dwarfs in open clusters mainly by Koester and Reimers (for details see Weidemann, Koester,1983, or Weidemann,1987c). For stellar evolution with intermediately strong overshooting, characterized by a parameter $\alpha_{\mathrm{C}}=0.5$ the upper mass limit for white dwarf production is about $M_{i} \approx 8 M_{\odot}$. Calculated M-distributions are broadened by a Gaussian with $\sigma=0.05 \mathrm{M}_{\odot}$ in order to account for observational errors. An essential result is reproduced in Fig. 4 (the influence of different IMFs turned out to be minor) which shows two distributions compatible with the observational histogram.

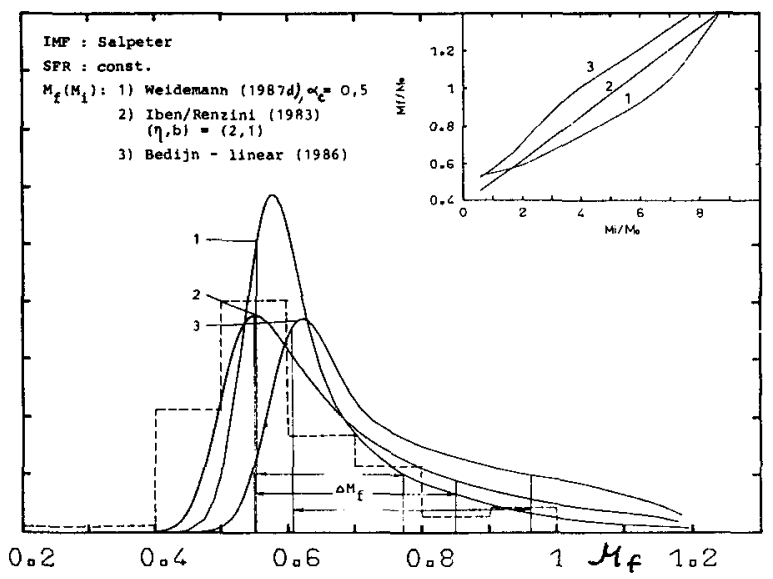

Fig.4. Mass distribution as calculated with galactic evolution model for different $M_{f}\left(M_{j}\right)$ relations (see insert);

1): Weidemann (1987d) $\alpha_{\mathrm{C}=0.5 \text {; }}$

2): Iben, Renzini (1983) for $(\eta, b)=(2,1),(\eta$ : Reimers wind loss factor, b: PN efficiency parameter);

3): Bedijn (1986) linearized relation derived from mass loss calculations for AGB evolution for OH/IR stars;

$\Delta M_{f}$ measures the $2 \sigma(67 \%)$ width;

---: DA distribution.

The left side indicates that either $M_{f}\left(M_{i}=1\right)$ should be assumed smaller, since relation 1 starts at $M_{f}=0.55 M_{\odot}$ implying that there are no white dwarfs with smaller masses, which is probably not true. The fraction of white dwarfs born below the PN visibility limit is at present unknown, it is composed of stars leaving the $A G B$ below it and of stars which do not reach the $A G B$ at all (horizontal branch stars, which enter the white dwarf region via sdo and $s d B$ channels). The fraction of the latter should not be larger than $10 \%$, although a firm estimate can be only be made for the extreme horizontal branch stars, with $0.45<\mathrm{M}_{c}<0.50 \mathrm{M}_{\Theta^{\prime}}$ for which Heber (1986) finds $2 \%$.

In this context it is worthwile to mention that the mass of $40 \mathrm{Er} i \mathrm{~B}$, for a long time considered to be well established by stellar dynamics, $(0.43$ $\pm 0.02 / \mathrm{M}_{\odot}$, is probably more around $0.50 \mathrm{M}_{\odot}$ as indicated by recent redshift observations of Wegner $(1980,1987)$ and Koester (1988).

The fraction of white dwarfs entering below the PN limit of $0.55 \mathrm{M}_{0}$ cannot be too large since otherwise the discrepancies between PN and WD birth rates, amounting to a factor of three (see Fleming et al., 1986) would become unbearable, although some remedy can be found by increased PN distances (Weidemann, 1988) and hidden white dwarfs in binaries. 
On the other hand there is evidence for differential mass loss at a given $\mathrm{M}_{i}$, thus the unique $\mathrm{M}_{\mathrm{i}} / \mathrm{M}_{\mathrm{f}}$-relations have to be replaced by some $k$ ind of strip (see Fig. 1 in Weidemann, 1987d). However, it is evident from the calculations that such a strip must be of restricted width in order to remain compatible with the observed narrow M-distributions.

As far as the results of Yuan's calculations are concerned it is impressive to show that one can rule out the $\mathrm{M}_{f}\left(\mathrm{M}_{i}\right)$-relation proposed by Mazitelli and D'Antona (1986b) which begins at $M_{f}=0.64 \mathrm{M}_{\odot}$ for $\mathrm{M}_{i}=1 \mathrm{M}_{\odot}$, although its slow increase results in an acceptable distribution, as far as its width is concerned.

The calculated M-distributions as presented comprise all white dwarfs above $\log \mathrm{L} / \mathrm{L}_{\odot}=-3.7$ corresponding to a cooling age of $2 \cdot 10^{9} \mathrm{yrs}$, down to $\mathrm{T}_{\text {eff }}=6000 \mathrm{~K}$ (Koester and Schönberner, 1986). Of course it is easy to obtain also the M-distribution at white dwarf birth which is identical to the M-distribution of CPN. Fig. 5 thus gives the predicted CPN M-distribution for a model which fits the white dwarf data well. It is unfolded and indeed appears compatible with the observed distribution.

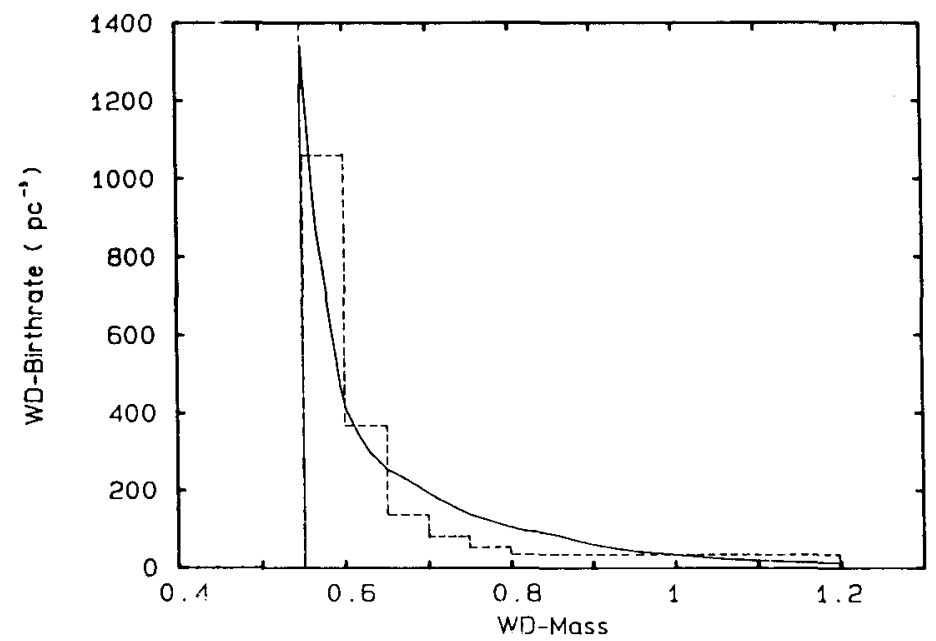

Fig.5. Predicted present WD birthrate or birthrate of $\mathrm{CPN}$, for galactic evolution model with IMF from Salpeter, $\mathrm{SFR}=$ const and $M_{f}\left(M_{i}\right)$ from Weidemann (1987c) for $\alpha_{C}=0.5$.

---: CPN mass distribution as derived from observations with the Schönberner method (local ensemble), cf. Weidemann (1988).

Yuan (1987b) has used the evolutionary tracks for CPN (Schönberner, 1981, 1983, Wood and Faulkner, 1986) to indicate the expected density of CPN in the HR-diagram, which is shown in Fig. 6.

It is evident that the true M-distribution can only be found by observing faint CPN since selection effects favor the observation of low mass CPN. A similar approach has been recently presented by Shaw (1988). However we have here included also $20 \%$ helium-burning CPN, which are found at higher luminosities (cf. Iben, 1984) so that the observed fraction at $\log \mathrm{L} / \mathrm{L} \approx 3$ should be about 508. Since WC type CPN are less frequent (17 - 20\%, Schönberner, 1986, Barlow, 1987) it appears that the $20 \%$ fraction assumed 


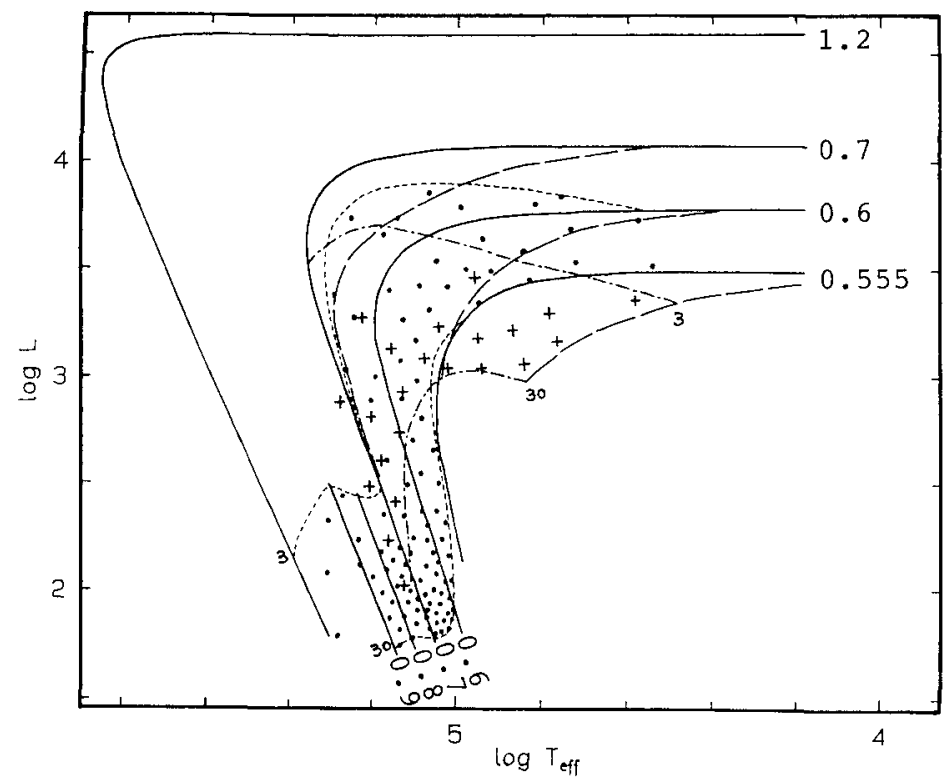

Fig.6. Expected density distribution of CPN in HR-diagram (Yuan, 1987b) with evolutionary tracks from Paczynski (1970), Wood and Faulkner (1986), and Schönberner (1981, 1983 ).

- : for hydrogen burning;

+ : for $20 \%$ helium burning CPN added;

- : evolutionary tracks for H-burning $\mathrm{CPN}$;

-- : for He-burning CPN.

Time interval

3000 to 30000 yrs

indicated by

--- for H-burning CPN, -.- for He-burning CPN.

is too high or that helium-burning stars retain a thin hydrogen atmosphere. PG 1159 objects, from their approximate location in the HR-diagram are probably helium-burning stars, which explains their relative frequency (see Weidemann, 1987a).

If we take all uncertainties into account we may close this section with the statement that $\mathrm{M}$-distributions of white dwarfs and planetary nebulae can be well understood within a comparatively simple model of galactic evolution.

\section{Possibilities of improvement}

As far as the observations is concerned, it is now possible to attain spectroscopy and photometry at higher signal to noise (see Greenstein, 1986) and higher resolution with modern detectors. High quality spectra like that of the DB star GD 358 (Koester et al., 1985) - enable more reliable analysis. In the case of DA stars, one should improve the $\mathrm{g} / \mathrm{T}$ eff determination by the analysis of high resolution line profiles, and by careful calibration, providing better $M(g)$. With Hipparcos coming up it can also be envisaged to obtain much better parallaxes, which are essential in order to determine $M(R)$ and to check on the mass-radius relation. The present situation is still completely unsatisfactory, as shown in Fig. 7, which gives the position of those DA stars whithin the wK 84 ensemble for which parallaxes are available. Some progress has recently been made by redshift determinations of high accuracy. (Wegner, 1987, 
Koester, 1987). I shall skip this since we shall hear more about it in Prof. Wegner's lecture (this volume).

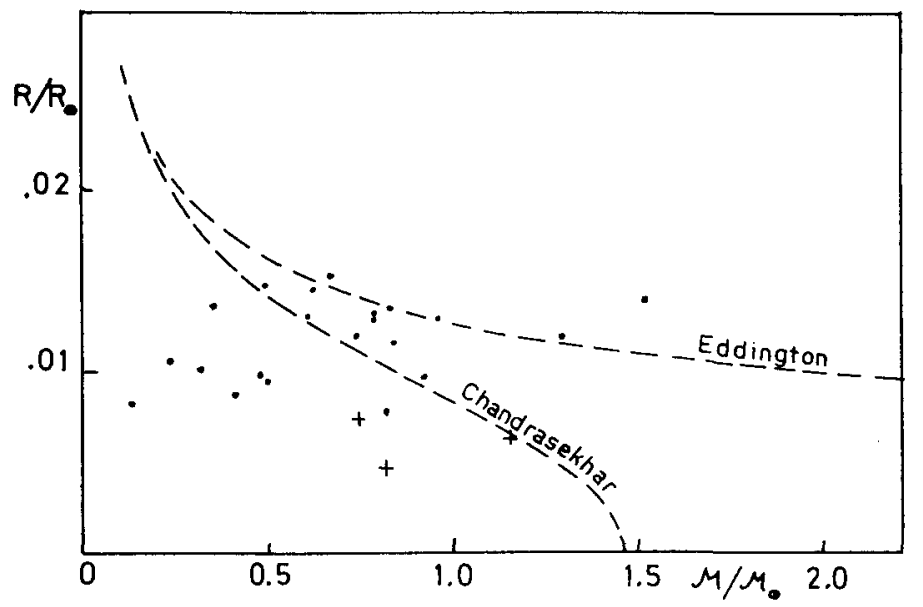

Fig.7. Mass and radii derived for 21 stars with known distances out of $70 \mathrm{DA}$ with well determined surface gravities (weidemann and Koester,1984), $M=M(R, g)$. If not for sirius $B$, at $1 M_{\odot}$, and three higher mass stars in NGC 2516 (WK 83 indicated by crosses, Chandra. sekhar would come out only marginally vs. Eddington.

In this context I should like to mention that the ongoing search for close double degenerates has yielded first results (cf. Saffer and Liebert, and the Bologna group, Bragaglia et al., this Conference). The smaller masses found for $L$ 870-2 (EG 11) are in line with predictions by Iben and Tutukov (1986) for a secondary peak in the number-mass distribution around $0.2-0.3 \mathrm{M}_{0}$, although the frequency of these objects is evidently smaller than first estimated.

I shall not go into details but refer to the other contributions concerning binarity at this conference.

On the theoretical side much improvement is needed in the field of stellar evolution which enters directly or indirectly many of our conclusions, e.g. concerning the presence of overshooting, the onset of the thermal-pulsing $A G B$ and mass loss mechanisms. Also models of galactic evolution should be extended to include different populations, and dependence on different locations in the Galaxy. Such population synthesis models have already been published (cf. Bienaymé et al., 1987) and white dwarf results shall appear if the proposed HST parallel survey - in which we participate - becomes reality.

Population II results are still too meager as to reach any conclusion. The local fraction should be of the order of a few percent (see sion et al., 1988). The exciting possibility to observe white dwarfs in globular clusters (first claimed by Richer and Fahlmann, this Conference) and to determine their masses will be also provided by the HST (see Renzini and Fusi Pecci, 1988). 
A final word should be said about selection effects. These have been shown to be extremely important in the case of CPN (see Heap and Augensen, 1987), and could have been influential also in the white dwarf case. This has been emphasized by Shipman (1979), taken up by Guseinov et al. (1983), but shown to be practically unimportant due to the very fact that the true M-dispersion is evidently narrow (Koester, 1984). But it is clear that for and with further improvements also selection effects have to be more carefully taken into account.

\section{The white dwarf luminosity function}

Since this topic has been covered recently by Liebert, Dahn and Monet (1988) and will be dealt with again in Liebert's following lecture (this Conference) I shall restrict myself to a few remarks and to the presentation of some relevant results obtained with the kiel galactic evolution program.

The discussion during the last years centered on three questions, which are closely connected, namely first: how is the shape of the LF at the cool end? Second: does the observed downturn of the LF reflect the finite age of the galactic disk and third: how reliable is the cooling theory, especially what is the influence of miscibility in the solid phase? To begin with the last question: the most recent contribution by Barrat, Hansen and Mochkovitch (1988) finds that the influence of a minor phase separation at crystallization on the WD LF is moderate and does increase the estimates of the age of the galactic disc from the downturn of the LF by winget et al. (1987) by only 0.50 to 0.75 Gyrs. However, for the second question, if the LF downturn measures the age of the galactic disk, there remain controversial statements. The calculations of Mazitelli and D'Antona, 1986a, predicted, and Larson's bimodal SFR (1986) needed a large number of WDs cooled down below invisibilty in order to explain the local missing mass.

On the other hand, it is important to notice that the population synthesis model by Bienayme et al. (1987) can explain the dynamical constants without the introduction of local missing mass, a result confirmed also by studies of the Cambridge group as recently presented at the Bologna ESO-CERN Conference by Lynden-Bell (1988).

Whereas Larson's hypothesis seems thus weakened the question of the extension of the cooling curves remains still important: as winget and Van Horn (1987) have demonstrated the raw ages of WDs cooled down to $\log \mathrm{L} / \mathrm{L}_{\odot}=-4.5$ range from 5 to 13 Gyrs according to different models and physical assumptions. 
The winget et al. (1987) conclusion for the galactic age must therefore be viewed with caution (especially as recent detailed studies of globular clusters by Buonanno et al. (1988) yield again a large age for the Galaxy, of $19 \pm 3$ Gyrs).

Its derivation has another weakness which lies in the fact that for the model calculations a constant WD birth rate (weighted by the DA M-distribution) has been assumed down to some time interval of 0.3 Gyrs after the beginning of the Galactic disk, which is estimated to be a mean pre-white dwarf lifetime.

Yuan 's calculations with our galactic evolution program however show that the results change significantly if the time dependence of the WD birth rate is taken into account.

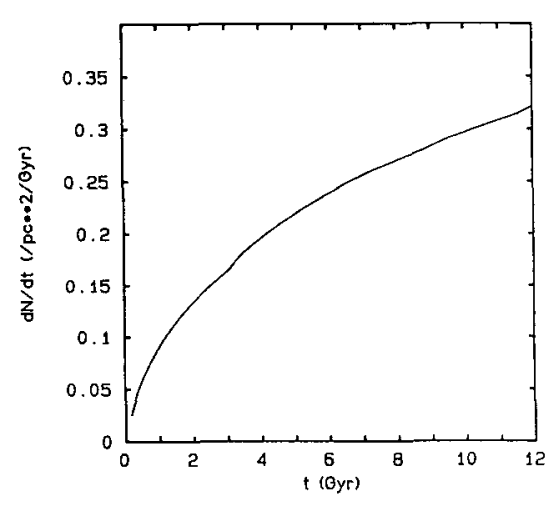

Fig.8. White dwarf birth rate increase as function of time. Galactic evolution model with IMF Salpeter, $S F R=$ const, $M_{f}\left(M_{i}\right)$ from Weidemann $(1987 c), \alpha_{C}=0.5$.

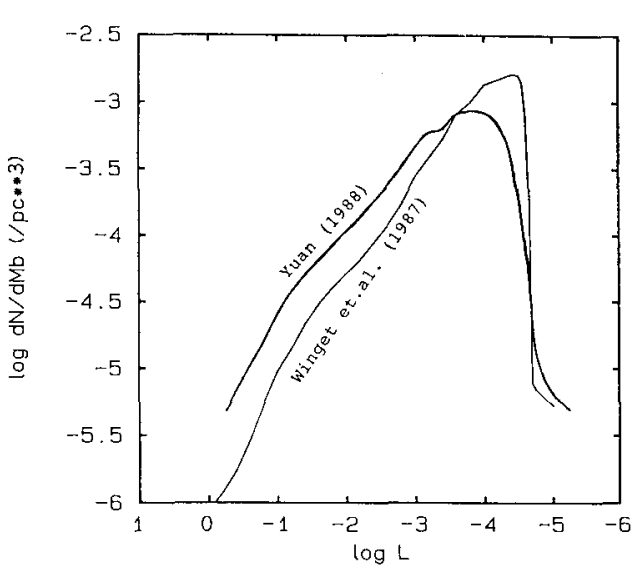

Fig.9. Luminosity function as calculated with galactic evolution model, compared to winget et al. modelling (see text).

Whereas Fig. 8 demonstrates how the wD birth rate increases gradually, Fig.9 shows the corresponding LF to run smoother and to display not such a steep downturn than in the winget et al. approach (keeping all other parameters as in their publication).

However it might be also interesting to show how the LF changes with other assumptions: on IMF - we can rule out the Larson model; on SFR we can demonstrate that it is not possible to conclude from the WD LF on $S F R(t)$ except in extreme cases of star bursts; on $M_{f}\left(M_{i}\right)$ - the differences are minor; on cooling curves and galactic age - it will be difficult to disentangle the effects, since longer or shorter cooling times can be nearly compensated by larger or smaller galactic ages. 
As always we thus have to conclude what is almost trivial: we need improvements as well on the observational side of very cool degenerates (cf. Ruiz and Anguita at this Conference) as on the interpretation of cool atmospheres and especially on cooling theory.

\section{References}

Barlow, M.J.: 1987, Mon.Not.Roy.Astr.Soc. 227, 161

Barlow, M.J.: 1988, in IAU Symp. No. 131, Planetary Nebulae eds. TorresPeimbert et al., in press

Barrat,J.L., Hansen,J.P., Mochkovitch,R.: 1988, Astron. Astrophys. 199, L15 Bedijn, P.J.: 1986 , preprint

Bienaymé, O., Robin, A.C., Crẻzé, M.: 1987, Astron. Astrophys. 180, 94

Buonanno, R., Corsi, C.E., Fusi Pecci, F.: 1988, Astron. Astrophys. submitted (ESO preprint No. 594)

Fleming, T.A., Liebert, J., Green, R.F.: 1986, Astrophys. J. 308, 176 Ford, H.C., Ciardullo, R., Jacoby, G.H., Hui, X.: 1988, in IAU Symp. No. 131. Planetary Nebulae, eds. Torres-Peimbert et al. in press

Fusi Pecci, F., Renzini, A.: 1976, Astron. Astrophys. 46, 447

Greenstein, J.L.: 1976, Astron. J. 81, 323

Greenstein, J.L.: 1984, Astrophys. J. 276, 602

Greenstein, J.L.: 1985, Publ. Astr. Soc. Pac. 97, 827

Greenstein, J.L.: 1986, Astrophys. J. 304, 334

Graham, J.A.: 1972, Astron. J. 77, 144

Guseinov,O.H., Novruzova,H.I., Rustamov,Y.S.: 1983, Ap. Space Sci. 96, 1 Habing, H.J.: 1988, Astron. Astrophys. 200, 40

Hayes, D.S., Latham, D.W.: 1975, Astrophys. J. 197, 593

Heap, S.R., Augensen, H.J.: 1987, Astrophys. J. 313, 268

Heber, U.: 1986, Astron. Astrophys. 155, 33

Hills, J.G., Dale, T.M.: 1973, Astrophys. J. 185, 937

Iben, I., Jr.: 1984, Astrophys. J. 277, 333

Iben, I., Jr., Renzini, A.: 1983, Ann. Rev. Astron. Astrophys. 21, 271

Iben, I., Jr., Tutukov, A.V.: 1986, Astrophys. J. 311, 753

Koester, D.: 1984, Astrophys. Space Sci. 100, 471

Koester, D.: 1987, Astrophys. J. 322, 852

Koester, D.: 1988, to be published

Koester, D., Schulz, H., Weidemann, V.: 1979, Astron. Astrophys. 76, 262

Koester, D., Weidemann, V.: 1980, Astron. Astrophys. 81, 145

Koester, D., Vauclair, G., Dolez, N., Oke, J.B., Greenstein, J.L.,

Weidemann, V.: 1985, Astron. Astrophys. 149, 423

Koester, D., Schönberner, D.: 1986, Astron. Astrophys. 154, 125

Larson, R.B.: 1986, Mon. Not. Roy. Astr. Soc. 218, 409

Liebert, J., Dahn, C.C., Monet, D.G.: 1988, Astrophys. J., in press 
Lynden-Bel1, D.: 1988, The Third ESO-CERN Symposium on Astronomy

Cosmology and Fundamental Physics, Bologna, in press

Mazzitelli, I., D'Antona, F.: 1986a, Astrophys. J. 308, 706

Mazzitel1i, I., D'Antona, F.: 1986b, Astrophys. J. 311, 762

Oke, J.B., Gunn, J.E.: 1983, Astrophys. J. 266, 713

Oke, J.B., Weidemann, V., Koester, D.: 1984, Astrophys. J. 281, 276

Reid, N., Mould, J.: 1985, Astrophys. J. 299, 236

Renzini, A., Fusi Pecci, F.: 1988, Ann. Rev. Astron. Astrophys. in press (Eso preprint No. 565)

Saffer, R.A., Liebert, J., Olszewski, E.W.: 1988, Astrophys. J. (Nov.)

Schönberner, D.: 1981, Astron. Astrophys. 103, 119

Schönberner, D.: 1983, Astrophys. J. 272, 708

Schönberner, D.: 1986, Astron. Astrophys. 169, 189

Schönberner, D., Weidemann, V.: 1983, in Planetary Nebuale, D. Flower ed., Reidel Dordrecht, p. 359

Shaw, R.A.: 1988, in IAU Symp. No. 131, Planetary Nebulae S. Torres-

Peimbert, M. Peimbert, J. Kaler eds. Reidel, in press

Shipman, H.L.: 1979, Astrophys. J. 228, 240

Sion,E.M., Fritz,M.L., MCMullin,J.P., Lallo,M.D.: 1988, Astron. J.

Wegner, G.: 1980, Astron. J. 85, 1255

Wegner, G.: 1987, in IAU Coll. No. 95. Second Conf.on Faint Blue Stars, A.G.D.Philip,D.S.Hayes, J.Liebert eds., L.Davis press, p. 649

Weidemann, V.: 1970, in IAU Symp. No. 42 White Dwarfs, W.J. Luyten ed.,

D. Reidel, p. 81

Weidemann, V.: 1977, Astron. Astrophys. 59, 411

Weidemann, V.: 1987a, IAU Coll.95, Second Conference on Faint Blue Stars. A.G.D.Philip, D.S.Hayes, J.W.Liebert eds., L. Davis press, p. 19

Weidemann, V.: 1987b, in Late Stages of Stellar Evolution. S. Kwok, S.R. Pottasch, eds. Reidel, p. 347

Weidemann, V.: 1987c, Astron. Astrophys. 188, 74

Weidemann, V.: 1987d, in 6th European Workshop on White Dwarfs. Mem. Soc. Astr. Ital. 58, 33

Weidemann, V.: 1988, Astron. Astrophys. submitted

Weidemann, V., Koester, D.: 1983, Astron. Astrophys. 121, 77

Weidemann, V., Koester, D.: 1984, Astron. Astrophys. 132, 195

Winget, D.E.,Van Horn, H.M.: 1987, IAU Coll.95, Second Conf.on Faint Blue Stars,A.G.D.Philip,D.S.Hayes, J.Liebert, eds. L.Davis press, p.363

Winget, D.E., Hansen,C.J., Liebert, J., Van Horn, H.M., Fontaine,G., Nather,R.E.,Kepler,S.O.,Lamb,D.Q.:, 1987, Astrophys. J. 315, L77

Wood, P.R., Faulkner, D.J.: 1986, Astrophys. J. 307, 659

Yuan, J.W.: 1987a, Diplomarbeit, Univ. Kiel

Yuan, J.W.: 1987b, Poster (Weidemann), IAU Symp. 101, Planetary Nebulae 